\title{
Pere Alsius y Torrent, prehistoriador *
}

\author{
Mariano ayarzaguena Sanz
}

Pere Alsius y Torrent (Banyoles, 1839 - 1915) fue un relevante personaje de la Cataluña peninsular del s. XIX, famoso por su actividad como prehistoriador e historiador local y comarcal.

En la presente comunicación expondré, como queda reflejado en el titulo, su importancia como prehistoriador y su labor en este campo.

Para comprender el trabajo de un investigador hay que tener en cuenta el ambiente en el que se desenvuelve y grupos a los que pertenece. Alsius se encontraba en la vanguardia del movimiento nacionalista cultural y científico que existia en pro de los estudios históricos y científicos de la Cataluña del s. XIx denominado La Renaixença, cuyo vehiculo impulsor era la lengua catalana. Su pertenencia a este movimiento lo podemos observar en: a) los temas que trata (históricos); b) uso frecuente de la lengua catalana; $c$ ) personas con las que se relaciona (Pujol y Camps, Botet y Sisó, Chía, Martorell, Sanpere y Miquel, etc.); y d) revistas en las que publica (Revista de Ciencias Históricas, La Renaxensa, Revista de Girona, Anuari de la Associació d'Excursions Catalana, Revista de la Asociación Artistico-Arqueológica Barcelonesa, etc).

Hernández Sanhauja (1868), entre 1860 y 1862, encontró un yacimiento prehistórico al excavar las murallas de Tarragona, pero sin darse claramente cuenta de lo que suponia sus hallazgos. Sánchez Comendador, en 1868, lee una memoria en la Universidad de Barcelona, por la que quiere demostrar la existencia del hombre durante el Cuaternario antiguo. Sin embargo, Alsius, a pesar de efectuar excavaciones y publi-

Trabajo presentado al "Congreso Internacional de Historia de los Pirineos, Cervera 1988 
caciones relativas a la prehistoria después de los anteriormente citados, ha sido considerado el iniciador de los estudios prehistóricos en la Cataluña española, por ser el primero en remontarse en sus estudios al Pleistoceno con el fin de buscar la más remota antigüedad de Cataluña, para lo cual realizó excavaciones en yacimientos prehistóricos. Debemos tener en cuenta que por su profesión de farmacéutico se encontraba instruido en Ciencias Naturales, y, en esos momentos, los estudios prehistóricos se encontraban dentro de ese campo.

Alsius y Bosóms realizaron, en la cueva denominada de la «Bora Gran d'en Carreras", excavaciones de gran transcendencia para el co. nocimiento del Paleolítico Superior en el Pirineo. Ésta era conocida desde que José Catá la hubiese explorado en 1866. Alsius la describió en 1871 por ver primera. (Alsius, 30-XI-1871).

Tras las investigaciones de Alsius, E. Harlé (1882) exploró la citada cueva buscando reno, que no se habia encontrado en las excavaciones de otras cuevas españolas, y que sin embargo resultaba frecuente en Francia. Encontró pequeñas laminillas de dorso que pensó que eran magdalenienses. Llevó el material a Mortillet quien las consideró como “incontestablemente magdalenienses".

Alsius (1895) reconoce idénticos tipos industriales entre los objetos que encontró en esta cueva y los que los franceses encuentran en el yacimiento de "la Madeleine». En 1908 volvió a incidir sobre esta cueva en la revista La Ilustración Catalana, publicación que no he podido ver.

Obermaier (1925) también cita la existencia de Aziliense y Cano Herrera (1987) de Solutrense.

Corominas cribará en 1929 la tierra de la escombrera producida tras la primera excavación de Alsius y publicó los resultados en 1949, previo estudio y publicación de Pericot (1945). Éste recuerda que es el único yacimiento magdaleniense que se conocía en la zona española de los Pirineos orientales en el momento de escribir su trabajo, en contraste con lo frecuente que resultaban en la zona francesa. Corominas especificó que se daria en esta cueva el Magdaleniense VI, V y IV del sistema de Breuil y un posible Tardenoisiense. Martin Almagro (1947) añade el Magdaleniense III. La no presencia del Magdaleniense I y II la podemos relacionar con la escasez de complejos industriales de estas fases en los Pirineos, según ha expuesto Clottes en este mismo Congreso.

La publicación de Corominas nos ofrece datos para deducir cómo se produjo la excavación durante la etapa de Alsius. El método consistía básicamente en la búsqueda de fósiles directores que pudieran datar el 


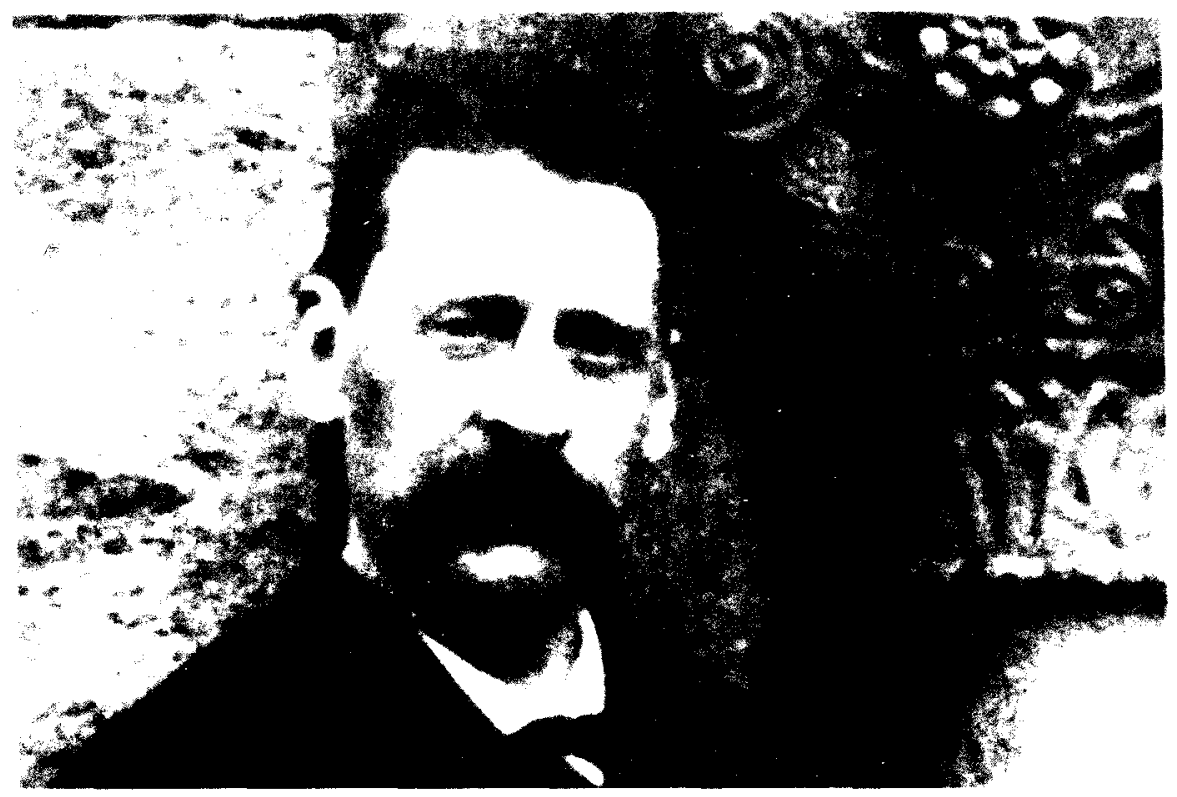

Pere Alsius y Torrent, (1839-1915).

yacimiento, olvidándose, la mayoria de las veces, de cribar las tierras que se extraian, y despreciando por consiguiente aquellos útiles de pequeño tamaño. Tenemos que tener en cuenta que las técnicas de excavación se encontraban muy influidas por el anticuarismo, encontrándose la ciencia prehistórica en un estadio incipiente.

A la vista de la publicación de Alsius (1895) podemos sacar conclusiones muy interesantes sobre cuáles fueron sus lecturas prehistóricas. Vemos que sigue con preferencia a los autores franceses: Mortillet, Cartailhac, Marqués de Nadaillac, Vernau, etc...; y escasamente lo que se realiza en el resto del estado español fuera de Cataluña; a este respecto nos consta la visita personal que le hicieron Cartailhac y Harlé. Una única vez cita a Vilanova, y no se trata de alguna de sus varias obras de conjunto, sino de una publicación en que daba cuenta de unos Congresos a los que habia asistido en el extranjero.

Resulta curioso que todavía en 1895 mantuviese las teorias catastrofistas relativas al Diluvio Universal, que por estas fechas ya se encontraban desfasadas en toda Europa, tras la general aceptación de las teorias fluvialistas de Lyell expuestas desde 1863. Esto nos lleva a pensar en lo limitado de sus lecturas prehistóricas y en la influencia de la Iglesia en este tipo de estudios en España. 
Otra cueva donde realizó excavaciones es la denominada de «Els Encantats", sacando a la luz sus investigaciones en la revista $L a$ Renaxensa (1871). Pensó que esta cueva funeraria se podia adscribir al Neolítico, pues, tal y como publica en 1895, no sabía de la existencia del hombre en la zona de su estudio durante la Edad de los Metales. A principios del presente siglo, Cazurro (1909) se ocupó de éste y otros yacimientos excavados por Alsius; pero más importantes fueron los estudios de Pericot (1945) y Maluquer (1948) sobre esta cueva. Pericot encontró restos cerámicos que él atribuyó a las épocas neolítica y hallstática. Maluquer establece, sin embargo, la existencia de un conjunto hallstático y de otro, más antiguo, relacionado con la cultura megalítica pirenaica. Estos autores descubrieron algunas piezas solutrenses tras el cribado de las tierras. Parece lógico que a Alsius se le escapara la industria solutrense esta época por el desconocimiento que existía de ella en España, pues la primera vez que fue citada en nuestro pais fue en 1891 por Vilanova.

El descubrimiento de la mandíbula neanderthaloide de Bañolas en el mes de abril de 1887 resulta un hallazgo de gran importancia. Dicha mandibula fue descubierta de manera fortuita, por un maestro cantero que estaba librando un pequeño bloque de toba con el fin de emplearlo como escalón. El prestigio de Alsius como historiador local fue, sin duda, lo que indujo a dicho trabajador a acudir a él con la pieza. Alsius, desde un primer momento, se dio cuenta de su gran antigüedad, aunque no se atrevió a adjudicarle una cronología.

Posteriores publicaciones que trataron de ella, como la de Cazurro (1909) o Harlé (1912), fueron en extremo escuetas. Hernández-Pacheco y Obermaier en 1915 verificaron un estudio más completo sobre la misma y la dataron como neandertaloide, aunque ya Cazurro (1909) la había relacionado, entre otras, con la de Spy, orientando la datación en ese camino. El célebre antropólogo español Hoyos (1947), también realizó un detenido trabajo anatómico sobre la citada mandibula, llegando a la conclusión de que se trataba de una "mandibula progresiva y final de la raza en el Musteriense superior». Marie-Antoinette de Lumley (1972) realizó un estudio exhaustivo y pormenorizado de la misma, señalando los antecedentes más importantes de los que la investigaron.

Fueron varios los trabajos y notas de Alsius enviados a diversas revistas especializadas y de información general, en los que bien realiza notas críticas sobre trabajos prehistóricos realizados por otros investigadores en Cataluña, bien realiza trabajos de conjunto.

En el primer caso, se puede citar la nota crítica sobre la publicación 
que Alfarás había realizado sobre descubrimientos prehistóricos que se habian efectuado en el Ampurdán (1897).

En el segundo caso cabe hacer mención especial del trabajo Serinyá y Caldas de Malavella (1882), donde establece la existencia de una tribu aborigen magdaleniense en Serinyá, y de otra tribu aborigen en Caldas, de la cual quedaron vestigios de la civilización neolítica. Dando por cierto que los monumentos megalíticos eran construcciones típicas del Neolítico, como era creencia común en el siglo pasado; al encontrar algunos dólmenes en las cercanías de Serinyá y de Caldas, deduce que se encontraban poblados ambos términos municipales en aquellos tiempos.

Sus conocimientos en Ciencias Naturales le resultan muy útiles en su faceta de historiador local y comarcal, tal y como podemos observar en su obra Ensaig histórich sobre la Vila de Banyolas (1872). En esta obra, con un espiritu que hoy resulta muy moderno, al realizar el estudio histórico, no se conforma con enumerar los conocimientos históricos que posee de la villa, sino que los antecede con datos de índole geográfico y geológico sobre dicha localidad; ya que entiende que los condicionamientos geográficos influyen, de alguna manera, sobre la evolución histórica de los pueblos.

Empieza la historia de la localidad, con la de sus más antiguos pobladores, es decir, con los prehistóricos, y no como solía ser costumbre, con los medievales, por to que se olvidaban de los primeros hombres que habitaron la zona, tal y como el propio Alsius denuncia.

En esta obra nos da a conocer las investigaciones arqueológicas que Francisco Martorell y Peña, José Gou, Manuel Bofill y el propio Alsius habian realizado al norte de dicha ciudad en el verano de 1871 . Se trata de unas tumbas excavadas en la roca, de diferentes formas y todas orientadas. Los huesos deben corresponder a los que la Comisión Provincial de Monumentos Históricos y Arqueológicos de Gerona en la sesión del 15-XI-1871 cita que se ingresó en el Museo de Gerona "un paquete de huesos humanos de unas sepulturas celtibéricas de junto a Bañolas".

Alsius opina que podria tratarse de tumbas de época celtibérica, en base a la cronología que atribuía Martorell (1879) a este tipo de sepulcros en la Peninsula lbérica. A este respecto escribía Martorell: «esta clase de sepulturas no se sabe que hayan sido usadas por los antiguos pueblos que han venido a España, como son los celtas, fenicios, griegos, cartagineses, romanos, suevos y visigodos. No habiendo podido traer estos pueblos el uso de las sepulturas olerdulanas, ¿á quiénes se puede referir 
mejor que a los iberos?». Alsius piensa también en la posibilidad de que sean en realidad de una época mucho más moderna. Debemos tener en cuenta el profundo desconocimiento que existía en esos momentos de lo que era el arte ibérico o celtibérico, frecuentemente confundido con el visigodo, error que se mantendrá hasta 1875 en que Rada describe el arte ibérico del Cerro de los Santos. Por nuestra parte, debido a la escasez de datos, no sabemos realmente a qué cultura podrían corresponder las citadas tumbas, aunque eran probablemente medievales.

Otra obra de Alsius, realizada conjuntamente con Celestino Pujol y Camps, premiada por la Asociación Literaria de Gerona en el certamen celebrado en 1882, es el Nomenclátor Geográfico-Histórico de la provincia de Gerona desde la más remota antigüedad hasta el siglo xv (1883). Aquí muestra las instituciones de la antigüedad que existian en Gerona, distinguiendo en el ámbito de su estudio una provincia histórica medieval de la actual, en plena línea de estudios de la Renaixença catalana.

Para terminar, queremos subrayar la transcendencia de su figura científica, ya que ayudó a divulgar los conocimientos prehistóricos en la Cataluña de este lado de los Pirineos. Asociado a esto, cooperó en la formación, fruto de su dinamismo, de una auténtica generación de prehistoriadores catalanes, como Martorell y Peña, Sanpere y Miquel, Chía, Pascual y Prats, Pujol y Camps, Conde de Bell-lloch, Texidor, Vayreda, Verdaguer, Vidal, etc., a quienes se les presentaron por primera vez los problemas que plantea la ciencia prehistórica en Cataluña y sobre cuya base se asentarán los Bosch Gimperá, Colomines, Corominas, Pericot, Serra Vilaró, Vilaseca, etc. 


\section{BIBLIOGRAFÍA}

Almagro Basch, M., 1947: El Paleolítico Español. Historia de España dirigida por Ramón Menéndez Pidal, tomo I, 1. España Primitiva. La Prehistoria, cap. III. págs. 243-286. Espasa-Calpe, Madrid.

ALSIUS Y TORRENT, P., 30-XI-1871. "Breu ensaig geológich de la conca de Banyolas". La Renaxensa, Any I, n. ${ }^{\circ} 21$, pág. 261-263. Barcelona.

- 1872: "Ensaig histórich sobre la vila de Banyolas". Folletí de la Renaxensa. Barcelona.

- 1882: "Serinyá i Caldas de Malavella". Anuari de l'Associació d'Excursions Catalana. Any segon. Barcelona, pág. 531-540.

- 1895: Serinyá: Reseña histórica de este pueblo desde la más remota antigüedad hasta los tiempos más modernos. Gerona.

- 1-III 1897: "Descubriments protohistorichs en l'Ampurdá». Revista de la Asociación Artístico-Arqueológico Barcelonesa, I, año $1 .{ }^{\circ}$, núm. 2 pág. 146. Barcelona. (Reproducción del publicado en el "Semanario de Bañolas").

- 1908: “El Magdalénico en Cataluña». Colección de articulos de La Ilustración Catalana. Barcelona.

Cano Herrera, M., 1987: "El Paleolítico Superior». Manual de Historia Universal, v. ${ }^{\circ}$ I. Prehistoria, págs. 163-218. Ed. Nájera. Madrid.

Cazurro y Ruiz, M., 1909: "Las cuevas de Serinyá y otras estaciones prehistóricas del NE de Cataluña". Anuari de l'institut d'Estudis Catalans II. Barcelona.

Corominas, J.M"., 1949: "La Colección Corominas de la "Bora Gran"» Monografías del Instituto de Estudios Pirenaicos, Fascículo III, Materiales Prehistoricos de Seriñá publicados bajo la dirección del $\mathrm{Dr}$. Luis Pericot Garcia. Zaragoza.

HARLE, E., 1882: "La grotte de Serinyá près de Gérone (Espagne). "Matériaux pour l'Histoire primitive et naturelle de l'Homme". XVIII année-2. ${ }^{\text {a }}$ serie, tomo XIII, págs. 293-299. 
- 1912: “Ensayo de una lista de Mamíferos y Aves del Cuaternario, conocidos hasta ahora en la Península Ibérica". Boletín del Instituto Geológico de España, tomo XXXII. págs. 135-162. Madrid.

Hernandez-Pacheco, E. \& Obermaier, H., 1915: “La Mandibula neandertaloide de Bañolas". Comisión de Investigaciones Paleontológicas y Prehistóricas, Memoria núm. 6. Museo Nacional de Ciencias Naturales. Madrid.

Hernandez Sanahuja, B., 1868: “Muros ciclópeos de Tarragona». Trabajo firmado en Tarragona el 1 de mayo de 1863. Memorias de la Real Academia de las Buenas Letras de Barcelona, tomo II, págs. 413-436. Barcelona.

Hoyos Sainz, L., de 1947: "Los Hombres Fósiles". Historia de España dirigida por Ramón Menéndez Pidal, tomo I, 1. "España Primitiva". La Prehistoria, cap. II págs. 117-144. En especial apartado 4. "La mandíbula de Bañolas", págs. 134-139, Madrid.

LUMLEY, M.A., de 1972: “La Mandibula de Bañolas". Ampurias, tomo 3334, págs. 1-91. Barcelona.

LyelL, SiR ChaRles., 1863: The Geological Evidences of the Antiquity of Man with Remarks on Theories of the Origin of Species by Variation. Londres.

MALUQUER DE MOteS, J., 1948: "Yacimientos postpaleolíticos". Materiales prehistóricos de Seriñá publicados bajo la dirección del Dr. Luis Pericot Garcia. Monografías de la Estación de Estudios Pirenaicos. Zaragoza.

Martorell y Peña, F., 1879: Apuntes Arqueológicos. Ordenados por Salvador Sanpere y Miquel. Barcelona

Obermaier, H., 1925: "El Hombre Fósil». Comisión de Investigaciones Paleontológicas y Prehistóricas, Memoria núm. 9, 2. ${ }^{a}$ Edición refundida y ampliada. Museo Nacional de Ciencias Naturales. Madrid.

Pericot Garcia, L., 1945: “Exploraciones arqueológicas en Seriñá (Gerona)». Pirineos I. págs 89-95. Jaca.

Pujol y Camps, C. \& Alsius y TorRent, P., 1883: Nomenclátor Geográfico-Histórico de la Provincia de Gerona desde la más remota antigüedad hasta el siglo XV.Gerona.

Rada y Delgado, J. de D. de la, 1875: Discursos leidos ante la Real Academia de la Historia en la recepción pública del Sr. D. Juan de Dios de la Rada y Delgado. Antigüedades del Cerro de los Santos en Término de Montealegre. Contestación de D. Aureliano Fernánder-Guerra y Orbe. Madrid.

SÁnchez Comendador y Pagniucci, A., 1868: Reseña histórica de los principales descubrimientos que realmente prueban la existencia del hombre durante el período cuaternario antiguo. Memoria leida ante el 
claustro de la Universidad de Barcelona el 29 de Noviembre de 1868 en la inaguración del Curso académico de 1868 a 1869. Barcelona.

VILANOVA Y PIERA, J., XII-1891: "Objetos protohistóricos de Arganda del Rey". Boletin de la Real Academia de la Historia vol. XIX C VI págs. 513-516. Madrid. 